\title{
Eficiência de fungos micorrízicos arbusculares isolados de solos sob diferentes sistemas de uso na região do Alto Solimões na Amazônia
}

\author{
Gláucia Alves e SILVA¹, José Oswaldo SIQUEIRA², Sidney Luiz STÜRMER³ \\ RESUMO \\ Os fungos micorrízicos arbusculares (FMAs) são importantes componentes dos ecossistemas terrestres onde acredita- \\ se desempenharem papel fundamental para a sustentabilidade destes. Estes fungos sofrem influência de diversos fatores \\ antrópicos como o uso da terra, que modificam a estrutura e diversidade das comunidades podendo comprometer suas \\ funçôes ecológicas. No presente estudo avaliou-se o comportamento de FMAs isolados de solos sob diferentes sistemas de uso \\ (SUT). Fungos isolados de amostras de solo sob diferentes SUT foram testados em caupi [Vigna unguiculata (L.) Walp] em \\ condiçōes controladas. Verificou-se que todos os cinqüenta e um fungos avaliados colonizaram o caupi, porém de modo muito \\ diferenciado, tal como ocorreu para os efeitos destes na absorção de fósforo e crescimento da planta. A colonização variou de \\ 1 a $68 \%$, e os efeitos positivos no crescimento variaram de 33 a 148\%, sendo mais comuns nos fungos isolados de pastagem \\ e roça. O aumento nos teores de fósforo foi generalizado ( $95 \%$ dos fungos testados), no entanto, nem todos foram capazes \\ de promover o crescimento do Caupi. Apenas 39\% dos fungos foram considerados eficientes, sendo estes isolados de quase \\ todos os SUT. Os tratamentos fúngicos de mais alta eficiência continham as espécies: A. foveata, Glomus sp.1, Acaulospora sp.1 \\ e mistura dos dois primeiros mais E. infrequens e A. bireticulata-like. Os resultados indicam ampla diversidade de eficiência \\ dos FMAs do Alto Solimões. Embora a eficiência não tenha relação direta com o SUT, a proporção de isolados eficientes \\ variou com a origem de isolamento.
}

PALAVRAS-CHAVE: simbiose radicular, fungos do solo, biodiversidade, cultivos amazônicos, recursos naturais.

\section{Effectiveness of arbuscular mycorrhiza fungal isolated from soils under different land use systems in the Alto Solimões river region in the Amazon}

\begin{abstract}
Arbuscular mycorrhizal fungi (AMF) are important components of terrestrial ecosystems where they are believed to play a fundamental role for their sustainability. These fungi are influenced by a number of anthropic factors such as, land use which modifies the structure and diversity of fungal communities and this may compromise their ecological functions. In the present study it was evaluated the behavior of AMF isolated from soils under different land use systems in (LUS). Fifty-one AMF were isolated in trap cultures from soil samples from different SUT tested on cowpea [Vigna unguiculata (L.) Walp] under controlled conditions. It was found that all AMF colonize the cowpea, but in a highly variable intensity. Similar results were found for phosphorus uptake and plant growth. Colonization ranged from 1 to $68 \%$. Growth positive effects ranged from 33 to $148 \%$, being more common in isolates from pasture and crops ecosystems. The enhancement in phosphorus uptake was generalized ( $95 \%$ all fungi), but their growth promoting effects did not followed the same trend. Only $39 \%$ of fungal isolated tested were efficient. These more isolated from almost all LUS. Fungal treatments with high efficiency had the following species: $A$. foveata, Glomus sp.1, Acaulospora sp. 1 and a mixture the first two species with $A$. bireticulata and $E$. infrequens. The results indicate a wide efficiency diversity of AMF in the Alto Solimões region. Although this characteristic has no direct relationship with the LUS, the proportion of effective isolates varied with their origin.
\end{abstract}

KEY WORDS: root symbiosis, soil fungi, biodiversity, amazonian cultivation, natural resources.

\footnotetext{
1 Universidade Federal de Lavras (UFLA), Departamento de Ciência do Solo (DCS), Caixa Postal 37, CEP 37200-000 Lavras-MG, Brasil. E-mail: glau_alvesilva@yahoo.com.br

2 Universidade Federal de Lavras (UFLA), Departamento de Ciência do Solo (DCS), Caixa Postal 37, CEP 37200-000 Lavras-MG, Brasil. E-mail: siqueira@cnpq.br

${ }^{3}$ Universidade Regional de Blumenau (FURB), Departamento de Ciências Naturais (DCN), Caixa Postal 1507, CEP 89012-900 Blumenau-SC, Brasil. E-mail: sturmer@furb.br
} 


\section{INTRODUÇÃO}

Os solos da Amazônia, quando submetidos ao desmatamento, perdem rapidamente a matéria orgânica, tornando-se inférteis (Cochrane \& Sanchez, 1982) e para se tornarem produtivos necessitam da reposição de nutrientes que geralmente não são disponíveis ou acessíveis aos produtores locais. Buscar alternativas para a fertilização destes solos é uma necessidade para conter a destruição da floresta e dentre as várias possibilidades, tem-se o emprego de agentes biofertilizantes como o rizóbio e os fungos micorrízicos arbusculares (FMAs). A obtenção de FMAs eficientes podem ser aplicados para incrementar o rendimento das culturas (Saggin-Junior \& Siqueira, 1996) e, assim, contribuir para a produção de alimentos na região.

Os FMAs ocorrem de maneira generalizada na natureza, colonizando cerca de $95 \%$ das espécies vegetais da maioria das famílias de plantas. Contudo, a distribuição das espécies fúngicas bem como a eficiência das mesmas são bastante variáveis e desuniformes, refletindo a ampla diversidade destes simbiontes e suas relações com as plantas hospedeiras e com o ambiente edáfico (Brundrett, 1991). O uso da terra, por exemplo, exerce efeito diferenciado sobre os FMAs, podendo causar modificação na estrutura das comunidades fúngicas, alterando a distribuição e dominância das espécies (Siqueira $e t$ al., 1989). Isto ocorre devido às alterações bióticas e abióticas do ambiente edáfico, como modificação das propriedades químicas do solo e da vegetação. Nos ecossistemas brasileiros já estudados, a riqueza de espécies de FMAs varia de 6 a 32, com média de 17 espécies por ecossistema (Stürmer \& Siqueira, 2006). Como os FMAs têm efeitos diferenciados sobre as plantas, os quais variam entre espécies e até mesmo entre isolados de uma mesma espécie, modificações nas comunidades destes fungos, em função de alterações no ecossistema podem ter conseqüências para as plantas hospedeiras (Dodd et al., 1990). Estas respostas diferenciadas resultam da complexa interação fungo-planta-ambiente (Smith \& Read, 1997), as quais são determinadas pelo grau de micotrofismo da planta e pela eficiência do fungo em estabelecer e promover o crescimento vegetal (Moreira \& Siqueira, 2006). Tem sido demonstrado que alteraçōes bióticas causadas pelo manejo do solo e das culturas influenciam a produção. Sieverding (1991) observou que a rotação de culturas com a mandioca resultou em aumento na proporção de esporos de isolados eficientes para esta cultura, de 33\% na monocultura para 52\% em sistema de rotação, incrementando, ainda, em duas vezes a produção de tubérculos de mandioca.

Apesar dos FMAs serem considerados sem especificidade hospedeira, há evidências de especificidade funcional quando se considera os efeitos destes fungos sobre a planta (PouyuRojas et al., 2006). Como as comunidades fúngicas variam de acordo com os ecossistemas, é importante avaliar as características destas comunidades, e em particular a eficiência de comunidades e de isolados que ocorrem em determinada condição, especialmente quando ecossistemas naturais sofrem interferências, como no cerrado do sudeste brasileiro (Siqueira et al., 1989) e em partes da Amazônia, onde a antropofização é intensa. Em termos práticos, a eficiência é caracterizada pela capacidade do fungo em colonizar amplamente e precocemente vários hospedeiros, favorecer a absorção de nutrientes do solo e transferi-los à planta, estimulando assim seu crescimento, desenvolvimento e produção (Abbott et al., 1992). Apesar de já terem sido desenvolvidos diversos trabalhos sobre as micorrizas arbusculares (MAs) na Amazônia (Bonetti, 1984; Moreira et al., 1997; Oliveira et al., 1997; Oliveira et al., 1999) estes se concentram na ocorrência de colonização em espécies florestais e efeitos da inoculação em espécies arbóreas nativas, empregando espécies fúngicas mantidas em coleçōes. Assim, pouco se conhece sobre a diversidade dos FMAs daquela região e seus efeitos no crescimento de plantas. Recentemente avaliou-se a ocorrência de espécies em diferentes sistemas de uso da terra (SUT) no Alto Solimões (Leal et al., 2009), estudo que revelou uma abundante e diversa comunidade de FMAs. Entretanto, a eficiência de FMAs isolados da Amazônia é ainda amplamente desconhecida. Dessa forma, conhecer a diversidade funcional dos FMAs da Amazônia é de grande interesse, diante do potencial destes fungos como alternativa para produção agrícola em áreas desmatadas da região.

Uma das culturas de grande importância na Amazônia é o feijão caupi [Vigna unguiculata (L.) Walp.], espécie que apresenta elevada suscetibilidade à colonização e resposta aos FMAs (Islan \& Ayanaba, 1981; Oliveira \& Bonetti, 1983, Almeida et al., 1985; Howeler et al., 1987; Raposo, 1989).

O presente trabalho é parte de um projeto mais amplo sobre a conservação e sustentabilidade da biodiversidade em solos tropicais, que objetivou avaliar a colonização micorrízica e a eficiência em feijão caupi, de FMAs isolados de solos da região do Alto Solimões, no Estado do Amazonas, sob diferentes sistemas de uso.

\section{MATERIAL E MÉTODOS}

O estudo constou de um experimento em casa de vegetação para avaliar a colonização e efeitos de 51 tratamentos fúngicos isolados de solos da Amazônia e previamente estabelecidos (ou multiplicados) em culturas armadilhas. As culturas armadilhas (sorgo e feijão caupi) foram estabelecidas misturando-se solo nativo (pastagem, roça, agrofloresta, capoeira nova e capoeira velha) com areia estéril $(1: 1, \mathrm{vol} / \mathrm{vol})$. Após seis meses, o solo foi retirado dos vasos e armazenado em câmara fria a $5^{\circ} \mathrm{C}$ até serem empregados na inoculação.

O experimento de inoculação dos FMAs isolados dos diferentes SUT foi conduzido em casa de vegetação do Departamento de Ciência do Solo da Universidade Federal de 
Lavras (DCS-UFLA) por 82 dias (início de floração do caupi). O solo utilizado para o crescimento do caupi foi um Latossolo Vermelho-Amarelo, muito argiloso, coletado na camada de 0-20 cm, em uma área de mata dentro do Campus da UFLA. O solo foi desinfestado com Bromex (98\% - brometo de metila $+2 \%$ - cloropicrina) na dose de $393 \mathrm{~cm}^{3} \mathrm{~m}^{-3}$ para eliminar propágulos de FMAs nativos. Posteriormente, o solo foi incubado com calcário dolomítico (PRNT 100\%), para elevar a saturação por bases e em seguida, foi realizada uma adubação básica com NPK, aplicando-se por kg de solo: $4 \mathrm{mg}$ de N, $7 \mathrm{mg}$ de P e $13 \mathrm{mg}$ de K. Posteriormente o solo foi acondicionado em tubetes de plástico com capacidade para $200 \mathrm{~cm}^{3}$ de solo para o plantio do caupi. Análises de fertilidade realizadas em amostras de solo após a calagem e adubação básica revelavam as seguintes características químicas: $\mathrm{pH}$ em água $=5,9 ; \mathrm{P}=$ $26,3 \mathrm{e} \mathrm{K}^{+}=181 \mathrm{em} \mathrm{mg} \mathrm{dm}{ }^{-3}$ (Mehlich 1); $\left(\mathrm{Ca}^{+2}=5,6 ; \mathrm{Mg}^{+2}=\right.$ 2,$\left.5 ; \mathrm{Al}^{+3}=0,0 ; \mathrm{H}^{+}+\mathrm{Al}^{+3}=2,1\right) \mathrm{em} \mathrm{cmol} \mathrm{dm}^{-3} ; \mathrm{V} \%=80,3 \mathrm{e}$ $\mathrm{MO}=3,8 \mathrm{dag} \mathrm{kg}^{-1}$.

Os tratamentos constaram de 51 tratamentos fúngicos isolados de solos sob diferentes (SUT) na Amazônia (Tabela 1), mais três tratamentos adicionais: uma mistura de todos os tratamentos fúngicos da Amazônia (Mi), inoculação com Glomus etunicatum Becker e Gerdemann (Ge), como uma referência para os tratamentos de inoculação, e uma testemunha não inoculada $(\mathrm{NI})$. A planta teste foi o caupi
[Vigna unguiculata (L.) Walp var. BR14 Mulato], que foi escolhida por ser uma espécie micotrófica e de interesse regional. Plântulas obtidas de sementes germinadas em papel de filtro, em câmera de germinação foram transplantadas para os tubetes, onde receberam os tratamentos de inoculação. A inoculação foi realizada pela adição de $50 \mathrm{~g}$ de solo inóculo abaixo da radícula das plântulas no ato da transferência de uma plântula por tubete. No tratamento sem inoculação, aplicaram-se $10 \mathrm{~mL}$ por tubete de um filtrado de inóculo sem propágulos de FMAs, mais $50 \mathrm{~g}$ do solo inóculo esterilizado duas vezes, para equilibrar as condições entre os tratamentos. Para o tratamento referência, aplicaram-se 100 esporos de G. etunicatum na radícula das plântulas por ocasião do transplantio. Os esporos de G. etunicatum foram obtidos de vasos de cultura da coleção da UFLA, por peneiramento úmido e centrifugação. Todos os tratamentos incluindo o NI foram inoculados com $1 \mathrm{~mL}$ de inoculante de rizóbio (Bradyrhizobium sp., estirpe INPA03-11B) por plântula. $\mathrm{O}$ experimento foi disposto em delineamento inteiramente casualizado, com 54 tratamentos, seis repetiçóes e uma planta por repetição. A irrigação foi feita de modo a manter a umidade do solo na capacidade de campo, com base no volume total de poros do solo, controlada por pesagens durante todo o período.

Tabela 1 - FMAs e número de esporos após multiplicação em Sorghum sudanense e Vigna unguiculata, e pH original das amostras de solo inóculo de diferentes sistemas de uso da terra, na região do Alto Solimões - AM.

\begin{tabular}{|c|c|c|c|}
\hline Tratamentos & FMAs encontrados (número esporos) & Esporos (por $50 \mathrm{~mL}$ de solo) & $\mathrm{pH}$ \\
\hline \multicolumn{4}{|l|}{ Pastagem } \\
\hline $82 P$ & A. foveata & 415 & 5,2 \\
\hline $84 \mathrm{P}$ & A. foveata & 143 & 5,1 \\
\hline $86 P$ & A. foveata & 206 & 5,3 \\
\hline $87 \mathrm{P}$ & A. foveata & 174 & 5,2 \\
\hline $89 \mathrm{P}$ & $\begin{array}{l}\begin{array}{l}\text { E. infrequens (10); } A \text {. bireticulata-like (4); Glomus sp.1 (1750); } A . \\
\text { foveata(45) }\end{array}\end{array}$ & 1809 & 5,2 \\
\hline $91 \mathrm{P}$ & Gigaspora sp (11); E. colombiana (283) & 294 & 5,3 \\
\hline $92 \mathrm{P}$ & A. foveata & 2115 & 4,9 \\
\hline $94 \mathrm{P}$ & Acaulospora tuberculata & 133 & 5,2 \\
\hline $95 \mathrm{P}$ & A. foveata & 270 & 5,2 \\
\hline \multirow[t]{2}{*}{$96 P$} & foveata (112); Archaeospora leptoticha (15) & 127 & 5,3 \\
\hline & \multicolumn{3}{|c|}{$127-2115$ * / 568,60** } \\
\hline \multicolumn{4}{|l|}{ Roça } \\
\hline $18 \mathrm{R}$ & Glomus sp.1 & 778 & 5,2 \\
\hline $19 \mathrm{AR}$ & Glomus sp.1 & 332 & 5,5 \\
\hline $21 \mathrm{R}$ & A. foveata (125); Glomus sp.1. (45) & 170 & 5,1 \\
\hline $26 \mathrm{R}$ & E. colombiana & 166 & 5,8 \\
\hline $28 \mathrm{R}$ & Glomus sp.1 & 156 & 4,9 \\
\hline $33 \mathrm{R}$ & Acaulospora sp.1 & 174 & 4,8 \\
\hline $44 \mathrm{~A} \mathrm{R}$ & Acaulospora sp.1 & 150 & 5,6 \\
\hline $49 \mathrm{R}$ & A. morrowiae (54); Glomus sp.1 (85); E. infrequens (4) & 143 & 5,1 \\
\hline $50 \mathrm{R}$ & Glomus sp.1 & 2060 & 5,1 \\
\hline
\end{tabular}


Tabela 1 - Continuação

\begin{tabular}{|c|c|c|c|}
\hline Tratamentos & FMAs encontrados (número esporos) & Esporos (por $50 \mathrm{~mL}$ de solo) & $\mathrm{pH}$ \\
\hline $51 \mathrm{R}$ & A. foveata & 110 & 5,3 \\
\hline $55 R$ & Glomus sp.1 (113); Acaulospora spinosa (2) & 115 & 5,2 \\
\hline \multirow[t]{2}{*}{$58 \mathrm{R}$} & Glomus sp.1 (311); Scutellospora sp. (1) & 312 & 6,2 \\
\hline & & $110-2060 / 388,83$ & \\
\hline \multicolumn{4}{|l|}{ Agrofloresta } \\
\hline $17 \mathrm{AF}$ & Glomus sp.1 & 246 & 4,8 \\
\hline $20 \mathrm{AF}$ & Paraglomus occultum (43) & 43 & 4,6 \\
\hline $24 \mathrm{~A} A F$ & Glomus sp.1 & 58 & 5,0 \\
\hline $25 \mathrm{AF}$ & A. morrowiae & 57 & 4,8 \\
\hline $66 \mathrm{AF}$ & morrowiae & 121 & 5,0 \\
\hline \multirow[t]{2}{*}{$67 \mathrm{AF}$} & A. morrowiae & 253 & 5,0 \\
\hline & & $43-253 / 129,67$ & \\
\hline \multicolumn{4}{|l|}{ Capoeira Nova } \\
\hline $01 \mathrm{CN}$ & Glomus sp. 1 & 206 & 4,4 \\
\hline $02 \mathrm{CN}$ & G. claroideum (140); G. clarum (54); E. infrequens (82) & 276 & 4,6 \\
\hline $29 \mathrm{CN}$ & Acaulospora delicata & 730 & 5,2 \\
\hline $30 \mathrm{CN}$ & Acaulospora delicata & 1850 & 4,4 \\
\hline $31 \mathrm{CN}$ & Acaulospora delicata & 650 & 5,0 \\
\hline $34 \mathrm{CN}$ & A. foveata & 308 & 4,9 \\
\hline $35 \mathrm{CN}$ & A. foveata & 3052 & 4,9 \\
\hline $38 \mathrm{CN}$ & Glomus sp.1 & 2186 & 4,3 \\
\hline $53 \mathrm{CN}$ & Glomus sp. 1 (612); Acaulospora sp. 1(143) & 755 & 4,6 \\
\hline $54 \mathrm{CN}$ & Glomus sp.1 & 370 & 4,7 \\
\hline $56 \mathrm{CN}$ & Glomus sp.1 & 138 & 5,8 \\
\hline $63 \mathrm{CN}$ & Glomus sp.1 & 252 & 5,4 \\
\hline $64 \mathrm{CN}$ & A. foveata & 170 & 5,0 \\
\hline $65 \mathrm{CN}$ & Glomus sp.1 & 51 & 5,1 \\
\hline $68 \mathrm{CN}$ & A. foveata & 2050 & 5,1 \\
\hline $69 \mathrm{CN}$ & A. foveata & 2810 & 4,7 \\
\hline $74 \mathrm{CN}$ & Glomus sp.1 & 60 & 5,2 \\
\hline \multirow[t]{2}{*}{$80 \mathrm{CN}$} & Acaulospora sp.1 & 115 & 5,0 \\
\hline & & $51-3052 / 890,50$ & \\
\hline \multicolumn{4}{|l|}{ Capoeira Velha } \\
\hline $23 \mathrm{CV}$ & Glomus sp.2 & 46 & 4,7 \\
\hline $37 \mathrm{CV}$ & Glomus sp.1 (1584); A. morrowiae (31) & 1615 & 4,8 \\
\hline $60 \mathrm{CV}$ & A. foveata & 150 & 5,6 \\
\hline $70 \mathrm{~A} C \mathrm{~V}$ & A. morrowiae & 360 & 4,7 \\
\hline \multirow[t]{2}{*}{$81 \mathrm{CV}$} & E. colombiana & 45 & 4,1 \\
\hline & & $45-1615$ / 443,20 & \\
\hline
\end{tabular}

*amplitude; **média.

Após 82 dias de crescimento, as plantas foram retiradas dos tubetes, separadas em parte aérea e raízes. As raízes foram lavadas e avaliadas quanto a nodulação e retirada aproximadamente $1 \mathrm{~g}$ de raízes frescas de cada planta para a coloração (Phillips \& Haymann, 1970) e a determinação da percentagem de colonização micorrízica (Giovanetti \& Mosse, 1980). Logo após, a parte aérea e raízes foram colocadas para secar em estufa com circulação forçada de ar, a $60^{\circ} \mathrm{C}$ até atingirem peso constante, em seguida, foram pesadas, obtendo-se a produção de matéria seca da parte aérea (MSPA), matéria seca de raízes (MSR) e matéria seca total (MSPA+MSR). A MSPA das plantas foi moída e analisada quimicamente quanto aos teores de $\mathrm{N}$ e P, conforme Zarosky \& Baurau (1977) e Malavolta et al. (1997).

A eficiência simbiótica (ES) dos fungos foi estimada a partir da produção da MSPA nos diversos tratamentos, aplicando-se a fórmula: 


\section{$\mathrm{ES}=[(\mathrm{I}-\mathrm{NI}) / \mathrm{NI})] \times 100$;}

onde I= MSPA de plantas inoculadas, NI= MSPA de plantas não inoculadas.

Todos os dados foram submetidos à análise de variância e teste de média (Scott-Knott, 5\%), utilizando-se o programa estatístico SISVAR (Ferreira, 2000), com os valores de colonização sendo previamente transformados em arco seno $(\mathrm{x} / 100)^{1 / 2}$.

\section{RESULTADOS E DISCUSSÃO}

\section{COLONIZAÇÃO MICORRÍZICA}

As plantas do tratamento testemunha não apresentaram sinais de colonização, ao contrário daquelas inoculadas nas quais a percentagem de colonização mostrou ampla variação entre os tratamentos fúngicos de 1 a $68 \%$ (Figura 1) e média geral de $30 \%$ de colonização. Ressalta-se que a maioria dos fungos apresentou boa colonização, com cerca de $70 \%$ dos tratamentos com colonização superior a $20 \%$. Com base nos níveis de colonização, os tratamentos fúngicos foram divididos em quatro grupos. O primeiro com os maiores níveis de colonização e média próxima de $60 \%$. Estes incluem as plantas inoculadas com os tratamentos fúngicos $60 \mathrm{CV}, 18$ R, 44A R, 55 R, 64 CN, 28 R, 17 AF, 37 CV, 35 CN, 29 $\mathrm{CN}$. Observa-se que estes foram isolados de amostras de solos provenientes dos mais variados sistemas de uso: agrofloresta, roça, capoeira nova e capoeira velha e as espécies Acaulospora foveata e Glomus sp.1 são encontradas em oito dos dez tratamentos com os maiores níveis de colonização (Tabela 1). Da mesma forma, estes fungos também estão presentes no grupo dos tratamentos que apresentaram as mais baixas taxas de colonização. Das espécies fúngicas estudadas, eles são os que aparecem com mais freqüência nos diferentes SUT. Os maiores níveis de colonização apresentados por este grupo não tem nenhuma relação com a densidade de esporos no inóculo. Um segundo grupo com colonização elevada, representado por 18 tratamentos, apresentou média de 35\% de colonização, seguido por outro grupo de 12 tratamentos, que exibiram colonização média de 19\%. Neste último grupo, também foi incluído o isolado G. etunicatum que apresentou colonização de apenas $16 \%$. Finalmente, um quarto grupo obteve os menores níveis de colonização, formado por 11 tratamentos mais a mistura de todos os FMAs da Amazônia (Mi), com valores médios de apenas $6 \%$ de colonização. Vários trabalhos têm mostrado elevado grau de colonização micorrízica do feijão caupi (Sieverding, 1991). Raposo (1989), trabalhando com duas variedades, obteve colonização acima de 60\%, enquanto Rohyadi et al. (2004), mesmo em condições de elevada acidez do solo ( $\mathrm{pH} 4,7)$, registraram colonização média superior a $35 \%$. Uma análise geral por SUT mostra efeito significativo da origem dos fungos na colonização (Tabela 2). A colonização obtida pelos fungos oriundos de roça e capoeira nova foi estatisticamente superior às encontradas nos demais SUT, os quais não diferiram entre si. Os resultados desta análise indicam que apesar da ampla variação entre os tratamentos, há efeito do sistema de uso, ou seja, fungos de diferentes origens após multiplicação em vasos apresentaram diferente capacidade de colonizar o caupi. A ampla variação ocorrida na percentagem de colonização dos tratamentos fúngicos avaliados pode estar sendo determinada pelas características dos isolados de FMAs que multiplicaram em cada amostra, como resultante da interação destes fungos com as condições específicas de ambiente e hospedeiro empregado na multiplicação (Koide, 1991). No entanto, não se observou qualquer relação entre a taxa de colonização e a densidade de esporos encontrada em cada cultura armadilha $(\mathrm{r}=0,21, \mathrm{p}>0,05)$, assim, como também no geral, não se verifica nenhuma relação da colonização com a composição de espécies dos tratamentos (Figura 1). Apesar de tratar-se de um teste preliminar que visa avaliar o comportamento de FMAs isolados de diferentes SUT, os resultados sugerem uma ampla diversidade de FMAs capazes de colonizar o feijão caupi, o que se reveste de grande interesse em termos de seleção e aplicação em programas de inoculação na Amazônia.

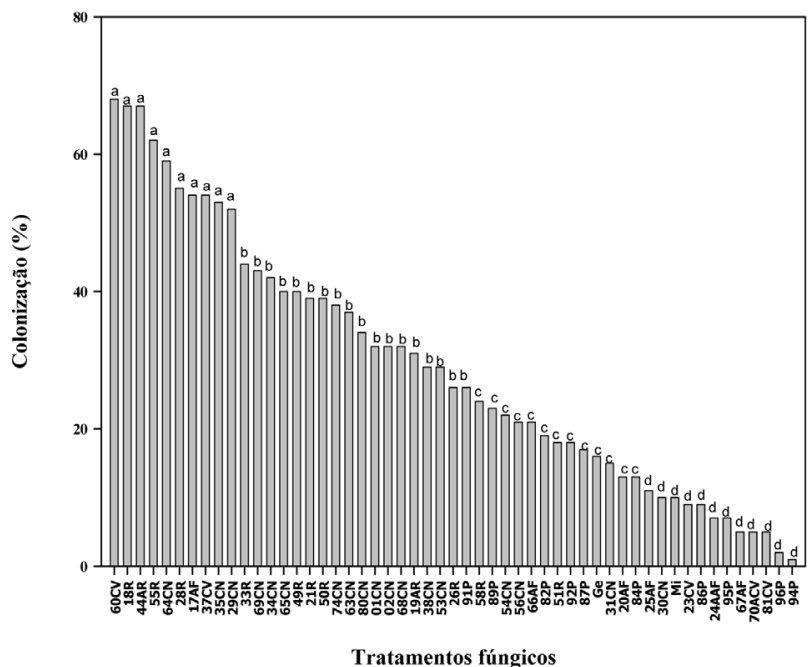

Figura 1 - Colonização micorrízica do caupi aos 82 dias após a inoculação com diferentes tratamentos de FMAs. Médias seguidas de letras iguais pertencem a um mesmo grupo pelo teste Scott-Knott 5\%.

\section{PRODUÇÃO DE MATÉRIA SECA}

A produção de matéria seca da parte aérea (MSPA), de raízes (MSR) e total (MSPA+MSR) foi influenciada significativamente pelos tratamentos de inoculação (Tabela 3). O percentual de tratamentos com efeito significativo na produção de MSPA do caupi em relação ao controle não inoculado (NI) foi de $76 \%$, sendo estes efeitos positivos no crescimento e encontrados em fungos isolados de todos 
Tabela 2 - Matéria seca da parte aérea (MSPA) e colonização micorrízica das plantas de caupi aos 82 dias após o plantio e inoculação com diferentes tratamentos de FMAs oriundos de diferentes sistemas de uso da terra na região do Alto Solimões-AM. Médias seguidas de letras iguais nas colunas pertencem a um mesmo grupo pelo teste Scott-Knott $5 \%$

\begin{tabular}{lccc}
\hline Origem dos FMAs & $\begin{array}{c}\text { Número de tratamentos } \\
\text { avaliados }\end{array}$ & $\begin{array}{c}\text { Colonização } \\
(\%)\end{array}$ & MSPA (g ) \\
\hline Pastagem & 10 & $13 \mathrm{~b}$ & $2,69 \mathrm{a}$ \\
Roça & 12 & $43 \mathrm{a}$ & $2,66 \mathrm{a}$ \\
Agrofloresta & 06 & $18 \mathrm{~b}$ & $1,87 \mathrm{~b}$ \\
Capoeira Nova & 18 & $34 \mathrm{a}$ & $2,44 \mathrm{a}$ \\
Capoeira Velha & 05 & $28 \mathrm{~b}$ & $2,11 \mathrm{a}$ \\
NI & ----- & ---- & $1,5 \mathrm{~b}$ \\
CV\% & & 43,04 & 29,46 \\
\hline
\end{tabular}

os sistemas de uso estudados. Fazendo uma análise geral por sistema de uso, verifica-se na tabela 2 , que apenas os tratamentos provenientes de agrofloresta não tiveram efeito médio significativo na produção de MSPA em relação ao controle NI. Entretanto, quando são analisados os efeitos isolados dos tratamentos (Tabela 3), tem-se um perfil bem diferente do que é revelado pela análise geral por sistema de uso. Verifica-se que todos os tratamentos de pastagem e roça, promoveram crescimento do caupi, e $78 \%$ daqueles da capoeira nova tiveram efeito significativo na produção de MSPA relativamente ao controle. Considerando os tratamentos originados de capoeira velha e agrofloresta, apenas $20 \%$ e $33 \%$ deles, respectivamente, aumentaram significativamente a MSPA. Os tratamentos que promoveram máxima produção de MSPA em cada sistema de uso foram: 87 P, 89 P, 92 P, 44A R, $35 \mathrm{CN}, 38 \mathrm{CN}$ e $60 \mathrm{CV}$, os quais proporcionaram incremento médio de $127 \%$, em relação ao NI. Nenhum tratamento de agrofloresta está presente dentro desse grupo que promoveu produção máxima, fato confirmado pela análise geral por sistema de uso (Tabela 2). Entre as amostras originadas de pastagem e roça, a proporção de tratamentos promovendo máxima produção de MSPA foi de $30 \%$ e $8 \%$, respectivamente. A inoculação com o Glomus etunicatum (Ge) e com a mistura dos tratamentos fúngicos dos diferentes SUT (Mi) proporcionaram aumentos na MSPA de $52 \%$ e $44 \%$, respectivamente. Deve-se ressaltar, contudo, que os efeitos na MSPA obtidos pela inoculação com o Ge, foram menores do que aqueles proporcionados por cerca de $50 \%$ dos tratamentos. Não se esperava que o Ge fosse superior, este foi empregado como referência por ser um fungo bastante comum em ecossistemas brasileiros e por ser um dos fungos mais comumente utilizados como isolados experimentais no Brasil (Stürmer \& Siqueira, 2006). A produção de MSR também foi influenciada pelos tratamentos, porém a incidência de tratamentos com efeito positivo foi de $57 \%$. Enquanto a totalidade dos fungos de pastagem e roça estudados estimulou a produção de MSPA, apenas $73 \%$ destes tiveram efeitos nas raízes (Tabela 3). Também os aumentos máximos na produção de MSR foram menos pronunciados que os encontrados para a MSPA. A análise para a produção

Tabela 3 - Matéria seca da parte aérea (MSPA), das raízes (MSR) e total (MSPA + MSR), teores de N e P na matéria seca da parte aérea das plantas de caupi aos 82 dias após o plantio e inoculação com diferentes tratamentos de FMAs oriundos de solos de diferentes sistemas de uso da terra, na região do Alto Solimões - AM. Médias seguidas de letras iguais nas colunas pertencem a um mesmo grupo pelo teste Scott-Knott 5\%

\begin{tabular}{|c|c|c|c|c|c|}
\hline \multirow{2}{*}{ Tratamentos } & MSPA & MSR & MSPA + MSR & $\mathrm{N}$ & $P$ \\
\hline & \multicolumn{3}{|r|}{ 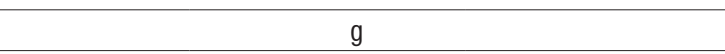 } & \multicolumn{2}{|c|}{$\mathrm{g} \mathrm{kg}^{-1}$} \\
\hline \multicolumn{6}{|l|}{ Pastagem } \\
\hline $82 \mathrm{P}$ & $2,74 \mathrm{~b}$ & $2,50 \mathrm{~b}$ & $5,17 \mathrm{a}$ & 20,83 e & $1,03 b$ \\
\hline $84 \mathrm{P}$ & $2,51 \mathrm{~b}$ & $2,37 \mathrm{~b}$ & $4,83 \mathrm{a}$ & 23,33 e & $1,07 b$ \\
\hline $86 P$ & $2,14 \mathrm{C}$ & $1,93 \mathrm{c}$ & $4,17 \mathrm{~b}$ & $21,96 \mathrm{e}$ & $0,34 d$ \\
\hline $87 \mathrm{P}$ & $3,72 \mathrm{a}$ & $2,47 b$ & $6,17 \mathrm{a}$ & $28,03 \mathrm{e}$ & $0,76 \mathrm{c}$ \\
\hline $89 \mathrm{P}$ & $3,27 \mathrm{a}$ & $2,06 \mathrm{c}$ & $5,33 \mathrm{a}$ & $21,56 \mathrm{e}$ & $0,66 \mathrm{c}$ \\
\hline $91 \mathrm{P}$ & $2,98 \mathrm{~b}$ & $2,16 \mathrm{~b}$ & $5,00 \mathrm{a}$ & $19,16 \mathrm{f}$ & $0,89 \mathrm{~b}$ \\
\hline $92 \mathrm{P}$ & $3,25 \mathrm{a}$ & $2,35 \mathrm{~b}$ & $5,33 \mathrm{a}$ & $22,70 \mathrm{e}$ & $0,78 \mathrm{c}$ \\
\hline $94 \mathrm{P}$ & $1,97 \mathrm{c}$ & $2,21 b$ & $4,33 \mathrm{~b}$ & $22,43 \mathrm{e}$ & $1,05 b$ \\
\hline $95 \mathrm{P}$ & $2,12 \mathrm{c}$ & $1,81 \mathrm{c}$ & $4,00 \mathrm{~b}$ & 20,76 e & $0,89 \mathrm{~b}$ \\
\hline $96 \mathrm{P}$ & $2,23 \mathrm{c}$ & $2,16 b$ & $4,33 \mathrm{~b}$ & $21,36 \mathrm{e}$ & $0,84 \mathrm{c}$ \\
\hline \multicolumn{6}{|l|}{ Roça } \\
\hline $18 \mathrm{R}$ & $2,95 \mathrm{~b}$ & $2,84 \mathrm{a}$ & $5,67 \mathrm{a}$ & $19,96 \mathrm{f}$ & $1,06 \mathrm{~b}$ \\
\hline $19 \mathrm{AR}$ & $2,07 \mathrm{c}$ & $2,05 \mathrm{c}$ & $4,33 \mathrm{~b}$ & $58,83 a$ & $1,44 \mathrm{a}$ \\
\hline $21 \mathrm{R}$ & $2,70 \mathrm{~b}$ & $1,66 \mathrm{c}$ & $4,17 \mathrm{~b}$ & $21,73 \mathrm{e}$ & $0,95 b$ \\
\hline $26 \mathrm{R}$ & $2,65 b$ & $1,95 \mathrm{c}$ & $4,33 \mathrm{~b}$ & $14,10 \mathrm{f}$ & $0,97 \mathrm{~b}$ \\
\hline $28 \mathrm{R}$ & $2,99 \mathrm{~b}$ & $2,26 b$ & $5,33 \mathrm{a}$ & 20,73 e & $1,16 \mathrm{a}$ \\
\hline $33 \mathrm{R}$ & $2,33 \mathrm{c}$ & $2,19 b$ & $4,50 \mathrm{~b}$ & $22,70 \mathrm{e}$ & $0,97 \mathrm{~b}$ \\
\hline $44 \mathrm{~A} \mathrm{R}$ & $3,56 \mathrm{a}$ & $2,28 \mathrm{~b}$ & $5,83 \mathrm{a}$ & $25,46 \mathrm{e}$ & $0,26 d$ \\
\hline
\end{tabular}




\begin{tabular}{|c|c|c|c|c|c|}
\hline \multirow{2}{*}{ Tratamentos } & MSPA & MSR & MSPA + MSR & $\mathrm{N}$ & $\mathrm{P}$ \\
\hline & \multicolumn{3}{|c|}{$\mathrm{g}$} & \multicolumn{2}{|c|}{$\mathrm{g} \mathrm{kg}^{-1}$} \\
\hline $49 R$ & $1,99 \mathrm{c}$ & $2,22 \mathrm{~b}$ & $4,17 \mathrm{~b}$ & $16,80 \mathrm{f}$ & $1,21 \mathrm{a}$ \\
\hline $50 \mathrm{R}$ & $2,55 b$ & $2,97 \mathrm{a}$ & $5,50 \mathrm{a}$ & $19,70 f$ & $0,83 \mathrm{c}$ \\
\hline $51 \mathrm{R}$ & $3,05 \mathrm{~b}$ & $2,13 b$ & $5,33 a$ & $22,30 \mathrm{e}$ & $0,98 b$ \\
\hline $55 \mathrm{R}$ & $2,48 \mathrm{~b}$ & $2,38 \mathrm{~b}$ & $4,83 \mathrm{a}$ & $17,86 f$ & $0,70 \mathrm{c}$ \\
\hline $58 \mathrm{R}$ & $2,62 \mathrm{~b}$ & $2,52 b$ & $5,00 \mathrm{a}$ & $16,36 f$ & $0,80 \mathrm{c}$ \\
\hline \multicolumn{6}{|l|}{ Agrofloresta } \\
\hline $17 \mathrm{AF}$ & $2,32 \mathrm{c}$ & $2,32 \mathrm{~b}$ & $4,83 a$ & $24,76 \mathrm{e}$ & $1,26 \mathrm{a}$ \\
\hline $20 \mathrm{AF}$ & $1,61 d$ & $2,23 b$ & $3,83 \mathrm{c}$ & 24,63 e & $1,31 \mathrm{a}$ \\
\hline $24 \mathrm{~A} A F$ & $1,86 d$ & $1,33 \mathrm{c}$ & $3,33 \mathrm{c}$ & $22,60 \mathrm{e}$ & $1,17 \mathrm{a}$ \\
\hline $25 \mathrm{AF}$ & $1,68 d$ & $1,39 \mathrm{c}$ & $3,00 \mathrm{c}$ & $22,90 \mathrm{e}$ & $1,36 \mathrm{a}$ \\
\hline $66 \mathrm{AF}$ & $2,14 \mathrm{c}$ & $1,87 \mathrm{c}$ & $3,83 \mathrm{c}$ & $45,33 \mathrm{c}$ & $1,10 b$ \\
\hline $67 \mathrm{AF}$ & $1,58 d$ & $1,55 \mathrm{c}$ & $3,17 \mathrm{c}$ & 31,53 e & $1,05 b$ \\
\hline \multicolumn{6}{|c|}{ Capoeira Nova } \\
\hline $01 \mathrm{CN}$ & $2,36 \mathrm{c}$ & $1,93 \mathrm{c}$ & $3,83 \mathrm{c}$ & $20,96 \mathrm{e}$ & $1,11 \mathrm{~b}$ \\
\hline $02 \mathrm{CN}$ & $1,83 d$ & $2,33 b$ & $4,33 \mathrm{~b}$ & $22,96 \mathrm{e}$ & $1,35 \mathrm{a}$ \\
\hline $29 \mathrm{CN}$ & $2,58 b$ & $2,87 \mathrm{a}$ & $5,50 \mathrm{a}$ & $37,56 \mathrm{~d}$ & $1,10 b$ \\
\hline $30 \mathrm{CN}$ & $1,72 d$ & $1,90 \mathrm{c}$ & $3,67 \mathrm{c}$ & $19,40 \mathrm{f}$ & $1,06 b$ \\
\hline $31 \mathrm{CN}$ & $2,30 \mathrm{c}$ & $1,49 \mathrm{c}$ & $4,00 \mathrm{~b}$ & $18,03 f$ & $1,07 b$ \\
\hline $34 \mathrm{CN}$ & $2,56 \mathrm{~b}$ & $2,61 \mathrm{a}$ & $5,17 \mathrm{a}$ & $18,83 f$ & $0,66 \mathrm{c}$ \\
\hline $35 \mathrm{CN}$ & $3,36 \mathrm{a}$ & $1,87 \mathrm{c}$ & 5,17 a & $15,46 f$ & $0,70 \mathrm{c}$ \\
\hline $38 \mathrm{CN}$ & $3,36 \mathrm{a}$ & $2,32 \mathrm{~b}$ & $5,67 \mathrm{a}$ & $20,03 f$ & $0,89 b$ \\
\hline $53 \mathrm{CN}$ & $2,99 \mathrm{~b}$ & $2,49 \mathrm{~b}$ & $5,50 \mathrm{a}$ & $15,86 f$ & $0,33 d$ \\
\hline $54 \mathrm{CN}$ & $2,95 \mathrm{~b}$ & $2,26 b$ & $5,50 \mathrm{a}$ & $17,50 \mathrm{f}$ & $0,67 \mathrm{c}$ \\
\hline $56 \mathrm{CN}$ & $1,60 \mathrm{~d}$ & $1,80 \mathrm{c}$ & $3,33 \mathrm{c}$ & $17,80 \mathrm{f}$ & $0,65 \mathrm{c}$ \\
\hline $63 \mathrm{CN}$ & $2,05 \mathrm{C}$ & $2,88 \mathrm{a}$ & $4,83 a$ & $18,86 f$ & $0,76 \mathrm{c}$ \\
\hline $64 \mathrm{CN}$ & $2,14 \mathrm{c}$ & $2,40 \mathrm{~b}$ & $4,67 \mathrm{a}$ & $25,36 \mathrm{e}$ & $0,92 b$ \\
\hline $65 \mathrm{CN}$ & $2,41 \mathrm{c}$ & $2,40 \mathrm{~b}$ & $4,83 a$ & 20,46 e & $1,09 b$ \\
\hline $68 \mathrm{CN}$ & $2,84 b$ & $2,03 \mathrm{c}$ & $4,83 a$ & $19,20 f$ & $0,94 \mathrm{~b}$ \\
\hline $69 \mathrm{CN}$ & $1,73 d$ & $2,04 \mathrm{C}$ & $3,67 \mathrm{c}$ & $17,60 \mathrm{f}$ & $1,00 b$ \\
\hline $74 \mathrm{CN}$ & $2,49 b$ & $1,96 \mathrm{c}$ & $4,50 \mathrm{~b}$ & 20,70 e & $1,00 \mathrm{~b}$ \\
\hline $80 \mathrm{CN}$ & $2,89 \mathrm{~b}$ & $2,44 \mathrm{~b}$ & $5,17 \mathrm{a}$ & $21,03 \mathrm{e}$ & $0,93 b$ \\
\hline \multicolumn{6}{|c|}{ Capoeira Velha } \\
\hline $23 \mathrm{CV}$ & $1,89 d$ & $1,97 \mathrm{c}$ & $4,00 \mathrm{~b}$ & $20,90 \mathrm{e}$ & $1,09 b$ \\
\hline $37 \mathrm{CV}$ & $1,87 d$ & $2,12 b$ & $4,17 \mathrm{~b}$ & $15,33 f$ & $1,04 b$ \\
\hline $60 \mathrm{CV}$ & $3,32 \mathrm{a}$ & $1,90 \mathrm{c}$ & $5,17 \mathrm{a}$ & $15,16 f$ & $0,82 \mathrm{c}$ \\
\hline $70 \mathrm{ACV}$ & $1,54 \mathrm{~d}$ & $2,10 \mathrm{c}$ & $3,67 \mathrm{c}$ & 20,96 e & $1,07 \mathrm{~b}$ \\
\hline $81 \mathrm{CV}$ & $1,93 d$ & $1,75 \mathrm{c}$ & $3,67 \mathrm{c}$ & $22,26 \mathrm{e}$ & $0,92 b$ \\
\hline $\mathrm{Ge}$ & $2,28 \mathrm{c}$ & $2,19 \mathrm{~b}$ & $4,33 \mathrm{~b}$ & $25,76 \mathrm{e}$ & $0,97 \mathrm{~b}$ \\
\hline $\mathrm{Mi}$ & $2,16 \mathrm{c}$ & $2,20 \mathrm{~b}$ & $4,33 \mathrm{~b}$ & $50,73 \mathrm{~b}$ & $0,94 b$ \\
\hline $\mathrm{NI}$ & $1,50 \mathrm{~d}$ & $1,76 \mathrm{c}$ & $3,67 \mathrm{c}$ & 20,96 e & $0,40 d$ \\
\hline CV\% & 23,26 & 20,50 & 17,49 & 25,03 & 25,93 \\
\hline
\end{tabular}

de MSPA+MSR revela efeitos diferenciados dos tratamentos. Em 78\% dos tratamentos estudados houve efeito na produção de MSPA+MSR e assim como na MSPA, estão distribuídos em amostras de todos os SUT (Tabela 3). Os efeitos da inoculação na MSPA+MSR foram mais generalizados e consistentes nos tratamentos originados de pastagem e roça, onde 100\% proporcionaram aumento na MSPA+MSR, com 60 e 58\% dos fungos isolados com efeito máximo, respectivamente. Os tratamentos que promoveram máxima produção de MSPA (87 P, 89 P, 92 P, 44A R, 35 CN, 38 CN e 60 CV), também proporcionaram máxima produção de MSPA+MSR, com incremento médio de 69\%. O efeito da inoculação com Ge e Mi, proporcionou aumento médio de 18\%. Os efeitos positivos da colonização micorrízica sobre a produção de 
matéria total obtidos neste trabalho confirmam o elevado grau de micotrofismo do caupi e sua resposta aos FMAs (Almeida et al., 1985; Rohyadi et al., 2004). Entretanto, os resultados revelam a enorme variação na capacidade dos fungos colonizarem e promover o crescimento desta cultura.

\section{TEORES DE NITROGÊNIO E FÓSFORO}

A inoculação influenciou também os teores de nutrientes no caupi, mas são apresentados aqui, apenas aqueles com os efeitos mais acentuados e consistentes, que ocorreram para o $\mathrm{N}$ e P na parte aérea (Tabela 3). Poucos tratamentos de inoculação tiveram efeitos significativos nos teores de $\mathrm{N}$. Os maiores teores de N na MSPA foram observados nas plantas inoculadas com os tratamentos Mi e 19A R, os quais proporcionaram incrementos de $142 \%$ e $181 \%$, respectivamente, em relação ao NI. A maioria das plantas inoculadas, entretanto, apresentou teores de N na MSPA iguais aos das plantas sem inoculação, ocorrendo em muitos casos, tratamentos fúngicos causando diminuição no teor desse nutriente nas plantas, e apenas quatro $(29 \mathrm{CN}, 66 \mathrm{AF}$, 19A R e Mi) deles elevaram os teores de $\mathrm{N}$ na MSPA, cujo incremento foi de 129\%, em média. Esse aumento, contudo, não se refletiu de modo positivo na produção de MSPA. Em nenhum dos tratamentos que proporcionaram produção elevada de MSPA teve também teores de $\mathrm{N}$ elevados nesta parte da planta. Isto, provavelmente, resultou do efeito de diluição, uma vez que estes tratamentos proporcionaram aumentos na MSPA. Efeitos de diluição nos teores de N em decorrência da micorrização são bem conhecidos em leguminosas: em soja, Paula et al. (1988) e outras espécies (Flores-Aylas et al., 2003). Em caupi, os maiores efeitos sobre a nutrição nitrogenada estão mais associados às plantas co-inoculadas com FMAs e bactérias fixadoras de nitrogênio (Almeida et al., 1984; Manjunath \& Bagyaraj, 1984). Os efeitos das MAs no fornecimento da nutrição nitrogenada é mais freqüente quando ocorre sinergia entre a micorrização e a nodulação o que não ocorreu no presente estudo. Apesar de inoculadas, por razões desconhecidas as plantas produziram poucos nódulos e de tamanho reduzido e assim, como não houve contribuição efetiva da fixação biológica de nitrogênio nas condições estudadas, os efeitos de diluição observados não representam surpresa. A razão para a reduzida nodulação está sendo investigada em outros estudos do grupo.

Os teores de fósforo na MSPA foram favorecidos pela inoculação com a grande maioria dos tratamentos testados e apenas os tratamentos 86 P, 44A R e $53 \mathrm{CN}$ não tiveram teores de P superiores ao controle NI (Tabela 3). O efeito da inoculação na absorção de fósforo pelo caupi no presente trabalho foi observado em condiçõos elevadas de P (26,3 $\mathrm{mg} \mathrm{dm}{ }^{-3}$ ), diferindo bastante dos valores de $\mathrm{P}$ encontrados nas áreas de onde os FMAs foram isolados, onde variam de 2,8 a 4,5 $\mathrm{mg} \mathrm{dm}^{-3}$ de P disponível (Nóbrega, 2006). Deve-se ressaltar, no entanto, que estes valores de P são níveis iniciais e que o volume de solo empregado no experimento foi muito pequeno. Interessa-se neste caso a quantidade de P disponível ao crescimento, passando-se o teor em si ter importância relativa. Os efeitos nos teores de $\mathrm{P}$ foram generalizados, ocorrendo em $95 \%$ dos tratamentos e atingindo incrementos de até $260 \%$ sobre as plantas sem inoculação. Os teores mais elevados de P na MSPA do caupi foram encontrados nas plantas dos tratamentos 19A R, $28 \mathrm{R}, 49 \mathrm{R}, 17 \mathrm{AF}, 20 \mathrm{AF}, 24 \mathrm{~A}$ $\mathrm{AF}, 25 \mathrm{AF}$ e $02 \mathrm{CN}$, atingindo valores de $1,44 \mathrm{~g} \mathrm{~kg}^{-1}$, quatro vezes maior que as plantas sem inoculação. Estes resultados são consistentes com outros estudos com caupi (Sanni, 1976, Islan et al., 1980; Almeida et al., 1984; Howeler et al., 1987; Wu et al., 2002; Rohyadi et al., 2004) em diferentes solos, condiçôes e fungos inoculados. Verifica-se que em $71 \%$ dos casos em que a inoculação aumentou a MSPA, houve também aumento nos teores de $\mathrm{P}$ nos tecidos, fato também observado por Siqueira \& Paula (1986) com soja, em solo pobre em P e inoculado com diferentes comunidades de FMAs. Também observa-se que os fungos eficientes, com exceção daqueles dos tratamentos $53 \mathrm{CN}$ e $44 \mathrm{~A} \mathrm{R}$, aumentaram a concentração de $\mathrm{P}$ em relação às plantas não inoculadas. Isto indica que estes fungos proporcionaram maior absorção de $\mathrm{P}$ e assim promoveram o crescimento do caupi nas condiçóes estudadas. Portanto, pode-se afirmar como tem sido demonstrado para a maioria dos efeitos das micorrizas sobre o crescimento das plantas, que os FMAs estudados promoveram o crescimento do caupi por facilitar a absorção de P do solo, reduzindo assim o déficit deste nutriente na planta (Siqueira, 1994). Entretanto, deve-se observar que em alguns casos houve aumento dos teores de P, sem o correspondente efeito no crescimento do caupi, evidenciando a influência de outros fatores além do déficit de P no crescimento das plantas nas condiçóes estudadas, conforme relatado em Stürmer (2004) trabalhando com soja. Pode ocorrer que o fungo seja capaz de promover o aumento na absorção de $\mathrm{P}$, mas apresenta alta demanda energética à planta comprometendo seu acúmulo de matéria seca.

Os tratamentos estudados representam um contínuo em relação aos efeitos na absorção de $\mathrm{P}$, variando de nenhum efeito ao máximo de $260 \%$. Isto sugere a existência de fungos ou comunidades muito eficientes em promover a absorção de $\mathrm{P}$ pelo caupi. Justifica-se a realização de estudos mais específicos e detalhados, objetivando conhecer os fungos mais eficazes, visando o manejo destes para a produção agrícola na região Amazônica.

\section{EFICIÊNCIA SIMBIÓTICA}

A eficiência simbiótica (ES) dos fungos estudados foi estimada pela capacidade destes em proporcionar acréscimos de MSPA nas plantas colonizadas em relação ao tratamento NI e a distinção entre fungos eficientes e ineficientes foi avaliado 
com base nas diferenças obtidas por teste estatístico (SkottKnott 5\%). A aplicação desta metodologia revelou que valores de ES inferiores ou iguais a $73 \%$ probabilisticamente não diferem do tratamento NI. Desse modo, apesar de um grande número de tratamentos terem aumentado à MSPA, apenas 20 deles (39\%) foram eficientes (Figura 2). Os tratamentos eficientes apresentaram variaçôes nesta capacidade, variando de 78 a $157 \%$. Analisando-se por origem, verifica-se que o maior número de fungos eficientes originou-se de roça e capoeira nova, seguido de pastagem. Tratamentos fúngicos eficientes foram encontrados em todos os SUT estudados, exceto em agrofloresta. A proporção de tratamentos eficientes nos diferentes SUT foi: $58 \%$ dos tratamentos de roça, $50 \%$ de pastagem, $39 \%$ de capoeira nova e $20 \%$ de capoeira velha. Do mesmo modo, tratamentos ineficientes foram encontrados em todos os SUT, porém com maior freqüência em agrofloresta, onde $100 \%$ dos tratamentos foram ineficientes (Figura $3)$. Dentre os fungos mais eficientes ( $>120 \%$ ), três são de pastagens (87 P, 89 P e $92 \mathrm{P}$ ), dois de capoeira nova ( $35 \mathrm{CN}$ e $38 \mathrm{CN}$ ), um de roça (44A R) e um de capoeira velha (60 $\mathrm{CV})$. Na maioria dos tratamentos mais eficientes (87 P, $92 \mathrm{P}$, $35 \mathrm{CN}$ e $60 \mathrm{CV}$ ) o fungo encontrado é a Acaulospora foveata e destes, os tratamentos $92 \mathrm{P} \mathrm{e} 35 \mathrm{CN}$ além de apresentarem a mesma espécie fúngica em comum, também têm o mesmo $\mathrm{pH}$ (Tabela 1). Este fungo também é encontrado em muitos dos tratamentos que foram ineficientes e é uma das espécies que aparece com mais freqüência no estudo, estando presente em todos os SUT, exceto em agrofloresta. Os resultados mostram que os tratamentos de alta eficiência não são encontrados em SUT específico; que a eficiência dos fungos estudados não se relaciona com a esporulação dos fungos em culturas $(\mathrm{r}=0,23, \mathrm{p}>0,05)$ e que o grau de colonização no caupi correlaciona-se positivamente com a eficiência fúngica $(\mathrm{r}=$ $0,40, \mathrm{p}<0,01)$. Observa-se na tabela 1 que o $\mathrm{pH}$ nas amostras de solo inóculo originais dos tratamentos que apresentaram alta eficiência variou de 4,3 a 5,6, dessa forma, é provável que a correção do solo para $\mathrm{pH}$ de 5,9 realizada na instalação do experimento tenha interferido na eficiência de algum isolado. É bom ressaltar, que estes fungos já haviam sido multiplicados em outro solo corrigido e mesmo com esta limitação os resultados permitiram boa discriminação comportamental, sendo válido como uma "screening" inicial de eficiência. Ficou evidente, a existência de FMAs com alta eficiência, sendo de grande interesse prático quando se deseja maximizar os efeitos benéficos desses fungos nativos sobre as plantas, especialmente daquelas de interesse para a região.

Esse trabalho indica que FMAs eficientes estão distribuídos aleatoriamente dentro do espaço geográfico ocupado por um sistema de uso da terra bastante dinâmico e diverso. Estudos mais abrangentes e profundos são necessários para conhecer e selecionar fungos eficientes, que possam ser utilizados em programas de inoculação de espécies de interesse prático para a produção na região Amazônica.

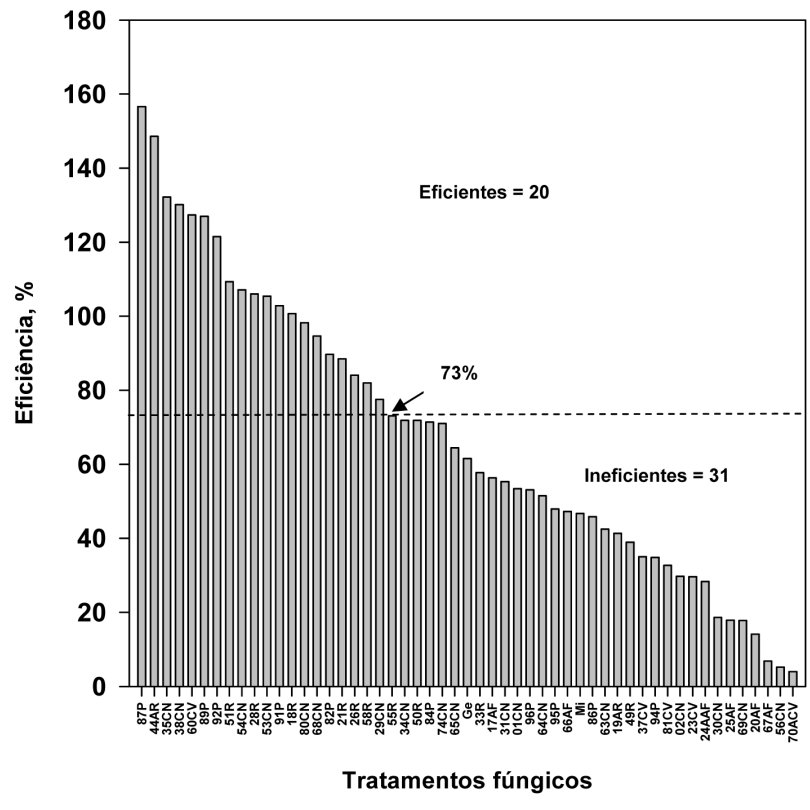

Figura 2 - Eficiência dos tratamentos fúngicos isolados de solos da Amazônia. A separação entre tratamentos fúngicos eficientes e ineficientes, feita com base nos resultados do teste de Scott-Knott $5 \%$, indica que tratamentos com valores de eficiência $\leq 73 \%$ são ineficientes por não diferirem de zero, correspondente ao controle não inoculado

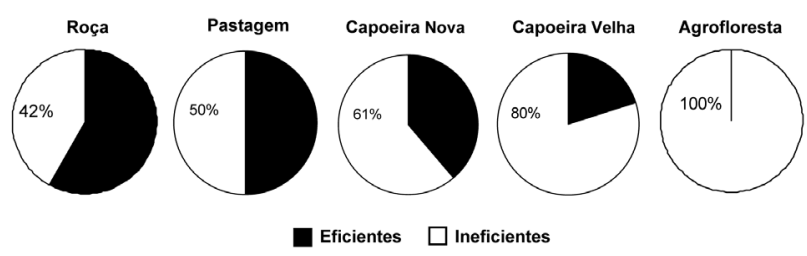

Figura 3 - Percentagem de tratamentos de FMAs eficientes e ineficientes nos diferentes sistemas de uso da terra na região do Alto Solimões-AM.

\section{CONCLUSÕES}

Os fungos isolados de solos da região do Alto Solimões - Amazônia sob diferentes sistemas de uso apresentam comportamento muito variado em relação à colonização, efeitos na absorção de P e no crescimento do feijão caupi.

Os parâmetros do comportamento simbiótico dos isolados revelam um contínuo de valores mínimos a valores elevados para todos os parâmetros estudados, indicando ampla diversidade funcional dos FMAs daquela região.

A capacidade de colonizar, absorver $\mathrm{P}$ e promover o crescimento do feijão caupi em condições controladas é generalizada dentre os fungos isolados, mas nem todos que colonizaram e forneceram a absorção de P são eficientes nas condiçōes estudadas. 
A proporção de fungos eficientes não é elevada (39\%) e é decrescente na seguinte ordem quanto à origem dos sistemas de uso dos quais foram isolados: roça e capoeira nova, pastagem, capoeira velha.

Os tratamentos fúngicos de mais alta eficiência continham as espécies: A. foveata, Glomus sp.1, Acaulospora sp.1 e mistura dos dois primeiros mais $E$. infrequens e $A$. bireticulata-like.

\section{AGRADECIMENTOS}

Esta publicação apresenta parte dos resultados do projeto internacional "Conservação e Manejo Sustentado da Biodiversidade do Solo" implementado em sete países tropicais - Brasil, Costa do Marfim, India, Indonesia, Quênia, Mexico e Uganda. Este projeto é coordenado pelo Tropical Soil Biology and Fertility Institute do CIAT (TSBF-CIAT) com co-financiamento do "Global Environmental Facility" (GEF), e suporte para implementação do "United Nations Environment Program" (UNEP). O componente brasileiro do projeto é denominado BiosBrasil e é coordenado pela UFLA (www.biosbrasil.ufla.br). As opiniōes expressas nessa publicação são de seus autores e não necessariamente refletem aquelas das instituições dos autores, do "United Nations Environment Programme" ou do "Global Environmental Facility."

\section{BIBLIOGRAFIA CITADA}

Abbott, L.K.; Robson, A.D.; Gazey, C. 1992. Selection Inoculant Vesicular-arbuscular Mycorrhizal fungi. Methods in Microbiology, 24(1): 1-21.

Almeida, R.T.; Ollivier, B.; Diem, H.G. 1984. Effect of Glomus mosseae and Pratylenchus sefaensis on growth of Vigna unguiculata. Ciência Agronômica, 15(1-2): 19-23.

Almeida, R.T.; Vasconcelos, I.; Sabadia, F.R.B. 1985. Efeito da infecção de fungos micorrízicos VA em feijāo-de-corda, Vigna unguiculata (L.) Walp. Ciência Agronômica, 16(1): 23-26.

Bonetti, R. 1984. Efeito de micorrizas vesiculares arbusculares na nodulação, crescimento e absorção de fósforo e nitrogênio em Siratro. Revista Brasileira de Ciência do Solo, 8(2): 189-192.

Brundrett, M.C. 1991. Mycorrhizas in natural ecosystems, In: Macfayden, A.; Begon, M. Fitter, A.H. (Eds). Advances in Ecological Research. vol. 21. London: Academic Press, p. 171313.

Cochrane, T.; Sanchez, P. 1982. Land resource, soil and their management in the Amazon region: a state of knowledge report. In: Hecht, S. (Eds). Amazon: agriculture and land use. Cali: Ciat. p.137-209.

Dodd, J.C.; Arias, I.; Koomen, I.; Hayman, D.S. 1990. The management of populations of vesicular-arbuscular mycorrhizal fungi in acid-infertile soils of a savannaecosystem II. The effect of pre-crops on the spore populations of native and introduced VAM-fungi. Plant and Soil, 122(2): 241-247.
Ferreira, D.F. 2000. Análises estatística por meio do SISVAR (Sistema para análise de variância) para Windows 4. 0. In: REUNIÃO ANUAL DA REGIÃO BRASILEIRA DA SOCIEDADE INTERNACIONAL DE BIOMETRIA, 45. 2000, São Carlos: UFSCar. p.255-258.

Flores-Aylas, W.W.; Saggin Junior, O.J.; Davide, A.C. 2003. Efeito de Glomus etunicatum e fósforo no crescimento inicial de espécies arbóreas em semeadura direta. Pesquisa Agropecuária Brasileira, 38(2): 257-266.

Giovannetti, M.; Mosse, B. 1980. An evaluation of techniques for measuring vesicular arbuscular mycorrhizal infection in roots. New Phytologist, 84(3): 489-500.

Howeler, R.H.; Sieverding, E.; Saif, S. 1987. Pratical aspects of mycorrhizal tecnology in some tropical crops and pastures. Plant and Soil, 100(1-3): 249-283.

Islan, R.; Ayanaba, A. 1981. Effects of seed inoculation and preinfecting cowpea (Vigna unguiculata) with Glomus mosseae on growth and seed yield of the plants under field conditions. Plant and Soil, 61(3): 341-350.

Islan, R.; Ayanaba, A.; Sanders, F.E. 1980. Response of cowpea (Vigna unguiculata) to inoculation with VA - mycorrhizal fungi and to rock phosphate fertilization in some unsterelized Nigerian soils. Plant and Soil, 54(1): 107-117.

Koide, R.T. 1991. Nutrient supply, nutrient demand and plant response to mycorrhizal infection. New Phytologist, 117(3): 365-386.

Leal, P. L.; Stürmer, S. L.; Siqueira, J. O. 2009. Occurrence and diversity of arbuscular mycorrhizal fungi in trap cultures from soils under different land use systems in the Amazon, Brazil. Brazilian Journal of Microbiology, 40(1): 111-121.

Malavolta, E.; Vitti, G.C.; Oliveira, S.A. 1997. Avaliação do estado nutricional das plantas: princípios e aplicaçôes. POTAFOS. Piracicaba. 201pp.

Manjunath, A.; Bagyaraj, D. J. 1984. Response of pigeon pea and cowpea to phosphate and dual inoculation with vesiculararbuscular mycorrhiza and Rhizobium. Tropical Agriculture, 61(1): 48-52.

Moreira, F.W.; Oliveira, L.A.; Becker, P. 1997. Ausência de micorrizas vesículo-arbusculares efetivas em Lecythidaceas numa área de floresta primária da Amazônia Central. Acta Amazonica, 27(1): 3-8.

Moreira, F.M.S.; Siqueira, J.O. 2006. Microbiologia e bioquímica do solo. 2. ed. Lavras, Minas Gerais: UFLA, 729pp.

Nóbrega, R. S. A. 2006. Efeito de sistemas de uso da terra na Amazônia sobre atributos do solo, ocorrência, eficiência e diversidade de bactérias que nodulam caupi [Vigna unguiculata (L.) Walp]. Tese de Doutorado, Universidade Federal de Lavras, Lavras, Minas Gerais. 188pp.

Oliveira, L.A.; Bonetti, R. 1983. Fatores químicos limitantes do solo e da nodulação, infecção por micorrizas VA e rendimento do feijão caupi num solo da região de Manaus. In: ENCONTRO DE PESQUISADORES DA AMAZÔNIA, 4. Porto Velho, Anais. Porto Velho- Rondônia. p.16. 
Oliveira, L.A.; Guitton, T.L.; Moreira, F.W. 1999. Relações entre as colonizaçôes por fungos micorrízicos arbusculares e teores de nutrientes foliares em oito espécies florestais da Amazônia, Acta Amazonica, 29(2): 183-193.

Oliveira, LA; Moreira, F.M.S.; Moreira, F.W. 1997. Ocorrências de microrganismos benéficos em ecossistemas amazônicos. In: Noda, H.; Souza, L.A.G.; Fonseca, O.J.M. (Eds). Duas décadas de contribuiçôes do INPA à pesquisa agronômica do Trópico Úmido. Manaus, INPA. p. 221-240.

Paula, M.A.; Siqueira, J.O.; Oliveira, L.H.; Oliveira, E. 1988. Efetividade simbiótica relativa em soja de populações de fungos endomicorrízicos nativos e de isolados de Glomus etunicatum e Gigaspora margarita. Revista Brasileira de Ciência do Solo, 12(1): 25-31.

Phillips, J.M.; Haymann, D.S. 1970. Improved procedures for clearing and staining parasitic and vesicular-arbuscular mycorrhizal fungi for rapid assessment of infection. Transactions of British Mycological Society, 55(1): 158-161.

Pouyú-Rojas, E.; Siqueira, J.O.; Santos, J.G.D. 2006. Compatibilidade simbiótica de fungos micorrízicos arbusculares com mudas de espécies arbóreas tropicais. Revista brasileira de Ciência do Solo, 30(3): 413-424.

Raposo, R.W.C. 1989. Inoculação de fungos micorrizicos vesículoarbusculares e Bradyrhizobium spp. em caupi [Vigna unguiculata (L.) Walp.J. Dissertação de Mestrado, Escola Superior de Agricultura Luiz de Queiroz, São Paulo, São Paulo. 84pp.

Rohyadi, A.; Smith, F.A.; Murray, R.S.; Smith, S.E. 2004. Effects $\mathrm{pH}$ on mycorrhizal colonisation and nutrient uptake in cowpea under contitions that minimise confounding effects of elevated available aluminium. Pant and Soil, 260(1-2): 283-290.

Saggin junior, O.J.; Siqueira, J.O. 1996. Micorrizas arbusculares em cafeeiro. In: Siqueira, J.O. (Eds). Avanços em fundamentos e aplicação de micorrizas. Universidade Federal de Lavras, Lavras, Minas Gerais. p.203-254.

Sanni, S.O. 1976. Vesicular-arbuslar mycorrhiza in some Nigerian soils and their affect on the growth of cowpea (Vigna unguiculata), tomato (Lycopersicon esculentum) and maize (Zea mays). New Phytologist, 77(3): 667-671.

Sieverding, E. 1991. Vesicular-arbuscular mycorrhiza management in tropical agrosystems. Eschborn: Friedland Bremer, 371 pp.

Siqueira, J.O. 1994. Micorrizas Arbusculares. In: Araujo, R.S; Hungria, M. (Eds). Microrganismos de importancia agricola. EMBRAPA: SPI. p. 151-194.

Siqueira, J.O.; Colozzi-filho, A.; Oliveira, E. 1989. Ocorrência de micorrizas vesicular-arbusculares em agro e ecossistemas do estado de Minas Gerais. Pesquisa Agropecuária Brasileira, 24(12): 1489-1498.

Siqueira, J.O.; Paula, M.A. 1986. Efeito de micorrizas vesículoarbusculares na nutrição e aproveitamento de fósforo pela soja em solo sob cerrado. Revista Brasileira de Ciência do Solo, 10: 97-102.

Smith, S.E.; Read, D.J. 1997. Mycorrhizal symbiosis. 2. ed. San Diego, California: Academic Press, 605 pp.

Stürmer, S.L. 2004. Efeito de diferentes isolados fúngicos da mesma comunidade micorrízica no crescimento e absorção de fósforo em soja e trevo vermelho. Revista Brasileira de Ciência do Solo, 28(4): 611-622.

Stürmer, S.L.; Siqueira, J.O. 2006. Diversity of Arbuscular Mycorrhizal Fungi in Brazilian Ecosystems. In: Moreira, F.M.S.; Siqueira, J.O.; Brussaard, L. (Eds). Soil Biodiversity in Amazonian and Other Brazilian Ecosystems. Londres, p.206-236.

Wu, T.; Hao, W.; Lin, X.; Shi, Y. 2002. Screening of arbuscular mycorrhizal fungi for the revegetation of eroded red sois in subtropical China. Plant and Soil, 239(2): 225-235.

Zarosky, R.J.; Burau, R.G. 1977. A rapid nitric perchloric acid digestion method for multi element tissue analysis. Communications in Soil Science and Plant Analysis, 8(5): 425436.

Recebido em 22/11/2007 Aceito em 27/04/2009 
